\title{
The presence of the marsupial Dromiciops gliroides in Parque Nacional Los Alerces, Chubut, Southern Argentina, after the synchronous maturation and flowering of native bamboo and subsequent rodent irruption
}

\author{
Yamila Gurovich ${ }^{1,2}$, Hayley J. Stannard ${ }^{3,4}$ and Julie M. Old ${ }^{3 *}$
}

\begin{abstract}
Background: Dromiciops gliroides is a small nocturnal marsupial found in the temperate forests of Southern Chile and Argentina. It is the only living member of the order Microbiotheria. Here, we describe the discovery and first account of live trapping of D. gliroides in Parque Nacional Los Alerces (Los Alerces National Park) in the province of Chubut, Argentina. This account extends the distribution of this cryptic species south to Parque Nacional Los Alerces (PNLA). The study provides a description of the habitat they were captured in after a recent bamboo flowering and seeding event and subsequent rodent irruption, the first in 70 years in PNLA.

Results: Four adult D. gliroides were captured during the study period, and both adult females captured had pouch young present, suggesting birth occurred in late October or early November. Habitat surveys revealed D. gliroides inhabited Nothofagus forest. A lack of fruiting plants in PNLA during the trapping period (spring) suggests their diet is likely restricted to insects at this time. No dreys were detected during habitat surveys, and hence, it is likely $D$. gliroides utilises tree hollows as refuges and nesting sites in PNLA. Significant threats to the population in PNLA were identified including introduced predators and human-related impacts due to farming and tourism.

Conclusions: There is a breeding population of D. gliroides present in Parque Nacional Los Alerces. The newly discovered population now represents the most southern location for D. gliroides in Argentina.
\end{abstract}

Keywords: Microbiotheria; Habitat surveys; Trapping; Camera traps; Patagonia; Marsupialia

\section{Background}

Monito del monte (Dromiciops gliroides) is a small nocturnal arboreal marsupial found in temperate forests in Chile (between $35^{\circ} 50^{\prime \prime} \mathrm{S}$ and $43^{\circ} 21^{\prime \prime} \mathrm{S}$ ) and southern Argentina (between $39^{\circ} 07^{\prime} \mathrm{S}$ and $42^{\circ} 00^{\prime} \mathrm{S}$ ) (Lobos et al. 2005; Amico et al. 2009; Celis-Diez et al. 2012). D. gliroides is the only living member of the order Microbiotheria (e.g. Nilsson et al. 2004), and more is closely related to Australasian marsupials than American marsupials (Kirsch et al. 1997; Palma and Spotorno 1999). It

\footnotetext{
*Correspondence: j.old@uws.edu.au

${ }^{3}$ Water and Wildlife Ecology, School of Science and Health, Western Sydney University, Penrith, NSW 2751, Australia

Full list of author information is available at the end of the article
}

is listed as 'near threatened' on the 2008 IUCN red list due to habitat destruction caused by logging and land clearing for agriculture (Diez and Teta 2008; Fontúrbel et al. 2010). As a frugivore, it likely plays an important role in seed dispersal (Amico and Aizen 2000; Amico et al. 2009; Rodríguez-Cabal and Branch 2011); however, it is primarily insectivorous (Mann 1955) and will eat insects, annelids, and on occasion small vertebrates (Jiménez and Rageot 1979; Celis-Diez et al. 2012).

In southern Argentina (Chubut province), D. gliroides is very rarely observed (Martin 2003). However, sightings of D. gliroides have been made in the province of Chubut including Parque Nacional Los Alerces (PNLA) and Parque Nacional Lago Puelo (Heinonen Fortabat 
and Chébez 1997). Due to these sightings, active trapping and infrared camera trapping was carried out in the spring and summer of 2014 in the PNLA, province of Chubut, Argentina.

Trapping occurred just after a synchronous bamboo flowering and seeding event which began in late 2012 in Villa Futalaufquen in the PNLA. The event continued along the Río Percy outside the PNLA, and was the first flowering and seeding event for the native bamboo in the PNLA in over 70 years. Native bamboo can take between 60 and 70 years to grow, flower and die (Pearson et al. 1994; Sage et al. 2007; Sanguinetti et al. 2012). This flowering event initiated a rodent irruption in the PNLA (beginning April 2013 and ending April 2014), and was likely similar to other rodent outbreaks that have previously occurred in southwestern Argentina (Sage et al. 2007). These rodent outbreaks are triggered by the abundance of bamboo seed with food availability normally the main limiting factor for rodents in these temperate forests.

This paper describes the results of live trapping and observations using cage and infrared camera traps to identify D. gliroides in the PNLA, Chubut, after a rare mass bamboo flowering and seeding event. It describes a new location for $D$. gliroides in the province of Chubut, Argentina, and extends the distribution range further south by approximately $40 \mathrm{~km}$ south to $42^{\circ} 43^{\prime} 47^{\prime \prime} \mathrm{S} 71^{\circ}$ $45^{\prime} 16^{\prime \prime} \mathrm{W}$, the southern-most site recorded for $D$. gliroides in Argentina. The paper also describes the habitat $D$. gliroides was located, and the threats posed to this population.

\section{Methods \\ Location}

The PNLA was created in 1937 to protect South America's largest endemic conifer Fitzroya cupressoides (Premoli et al. 2000). It lies between $42^{\circ} 50^{\prime}$ $40.3^{\prime \prime} \mathrm{S}$ and $71^{\circ} 50^{\prime} 17.4^{\prime \prime} \mathrm{W}$ in the subantarctic region (Cabrera 1971) of Chubut province and incorporates 263,000 ha, of which 187,500 ha is a national park and the remainder a national reserve (APN 1997).

The native vegetation in these forests is dominated by conifers (Austrocedrus chilensis and Fitzroya cupressoides), and beech trees (Nothofagus dombeyi, Nothofagus pumilio and Nothofagus antarctica). The understorey is dominated by native bamboo (Chusquea culeou) and shrubs including maqui (Aristotelia chilensis) and prickly heath (Gaultheria spp.).

The PNLA has a mean annual temperature of $8{ }^{\circ} \mathrm{C}$ and has an annual precipitation between 800 and $3000 \mathrm{~mm}$, mainly falling in April to October. Snow falls from June to September but can fall as late as October (APN 1997).

\section{Cage and camera trapping surveys}

In the PNLA, wire cage and infrared camera trapping was conducted from October to early December 2014. Trapping was conducted in three locations (A, B and C; Fig. 1). The first trapping location (A, Mermoud), is the ex-Mermoud settlement (no longer a settlement) on the shores of the Lago (Lake) Verde, near Puerto (Port) Mermoud which includes building ruins, animal yards, holding pens surrounded by orchards and introduced plants $\left(42^{\circ} 43^{\prime} 21^{\prime \prime} \mathrm{S} 71^{\circ} 45^{\prime} 02^{\prime \prime} \mathrm{W}\right)$. The settlement is adjacent to native old-growth forest areas. The second location (B, Río Menéndez) extends from the Pasarella (footbridge) over Río Arrayanes, along the path to Puerto Chucao in the Nothofagus forest bordering the Río Menéndez, and along the track to Puerto Mermoud $\left(42^{\circ} 43^{\prime} 43^{\prime \prime} \mathrm{S} 71^{\circ} 44^{\prime} 50^{\prime \prime} \mathrm{W}\right)$. The third location (C, Puerto Sagrario) was the old growth alerce (Fitzroya cupressoides) forest at Puerto Sagrario, which borders both Lagos Menéndez and Cisne and is only accessible by boat. No cage trapping was conducted at location $C$ due to limited accessibility $\left(42^{\circ} 36^{\prime} 38^{\prime \prime} \mathrm{S} 71^{\circ} 53^{\prime} 27^{\prime \prime} \mathrm{W}\right)$.

Cage traps were placed at random throughout locations A, B and C; however, placement was influenced by accessibility. Traps were placed along tree branches, approximately $1.5 \mathrm{~m}$ off the ground, and camera traps were placed in the same area with the aim of maximising detectability of small arboreal mammals.

\section{Habitat surveys}

Standard habitat surveys were conducted to allow a comparison between different cage and camera trapping sites $(n=19)$. A list of the GPS locations for each of the 19 sites is provided in Table 1. Habitat characteristics were measured at 10,25 and $40 \mathrm{~m}$ along the $50-\mathrm{m}$ line transect including percentage canopy cover, shrubs (0.5$2 \mathrm{~m})$, shrubs $(2-4 \mathrm{~m})$, herbage, rocks/logs and litter, within a $10-\mathrm{m}$ radius of the transect. In addition, plant species in the transects were identified, and dominant trees, shrubs and ground covers recorded using Dimitri (1977), Rapoport et al. (2003), Bisheimer and Fernández (2009), and Hoermann (2013). All trees with a diameter at breast height over bark larger than $10 \mathrm{~cm}$ were recorded. Any evidence of vertebrates in the survey site was recorded, including scats, scratchings and diggings. If any weeds were present, they were recorded, as well as the elevation and distance to water of each site.

\section{Trapping}

Trapping was conducted using custom-made wire cage traps $(26 \times 13 \times 13 \mathrm{~cm})$ during October, November and early December 2014. Traps were baited with either banana, apple, a combination of apple and banana, or bait balls (oats, honey and peanut butter) and set at dusk. In total, 778 trap nights were conducted over the trapping 


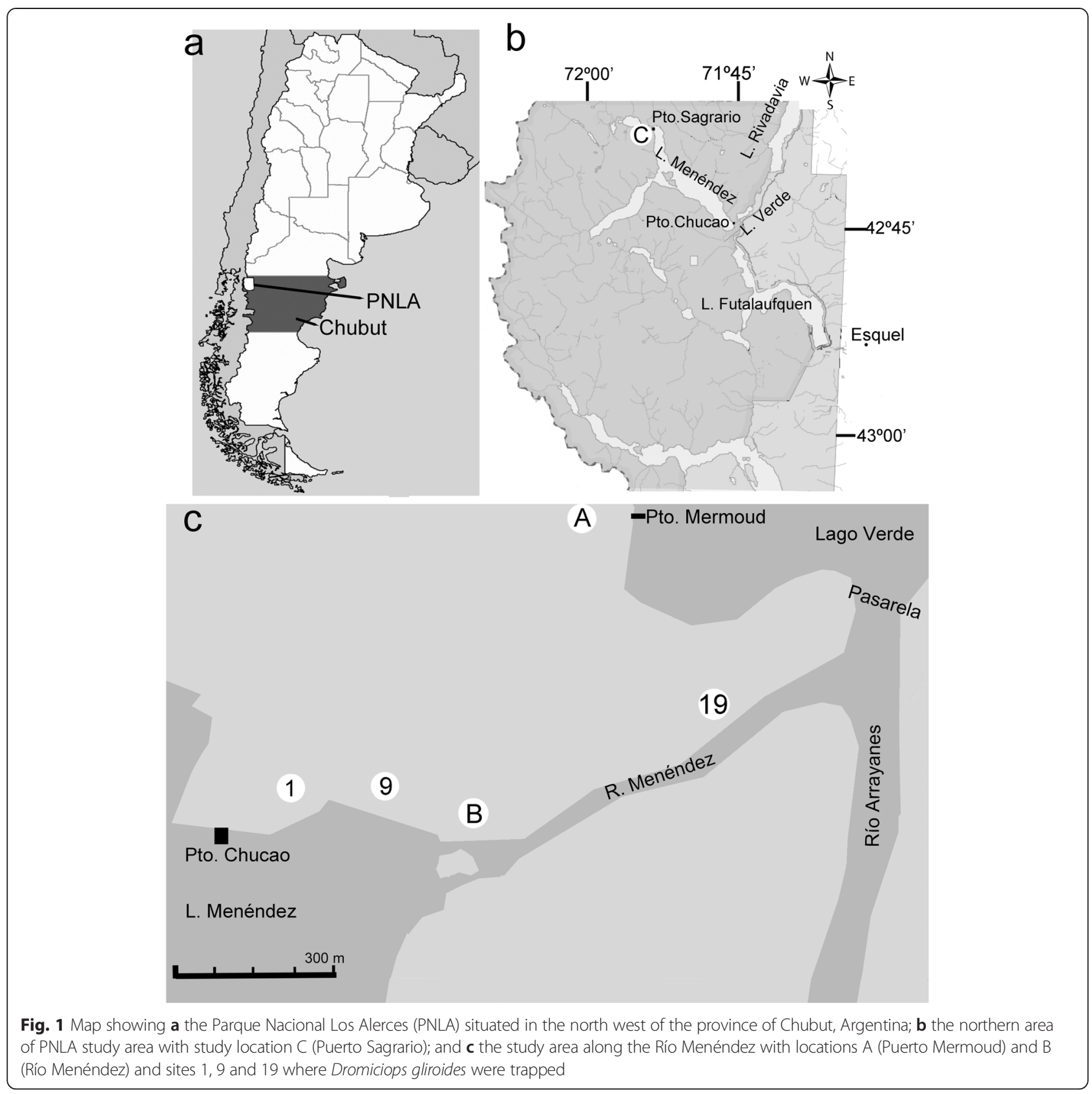

period. All traps were placed $0.5-2 \mathrm{~m}$ from the ground on mostly horizontal branches. All traps were opened at dusk and checked within $2 \mathrm{~h}$ of sunrise the following day. If traps were open in the morning, they were closed to prevent birds entering during the day.

\section{Infrared camera trapping}

Sixteen Range Ops XR mini infrared camera (Browning Trail Cameras, Birmingham, AL, USA) traps were placed on trees at the sites where cage traps were placed (see Table 1 for GPS locations), from October to midDecember inclusive. Cameras were placed on trees at approximately $1-1.5 \mathrm{~m}$ from the ground. Cameras recorded video when motion was detected. Cameras were set to take 30-s videos with a 30-s break between each video. Cameras also recorded temperatures, time and date when triggered.

\section{Sex and morphological data collection}

Traps were checked within $2 \mathrm{~h}$ of sunrise, and any animals captured offered water prior to examination. $D$. gliroides were examined to measure morphological characteristics, determine sex and assess health condition. All animals were weighed using a spring scale (Pesola, 
Table 1 GPS location for each cage and camera trapping site. The number of cage and camera trap nightsper site is also provided

\begin{tabular}{|c|c|c|c|}
\hline Site number & GPS location & $\begin{array}{l}\text { Wire trap nights } \\
\text { per site }\end{array}$ & $\begin{array}{l}\text { Camera trap } \\
\text { nights per site }\end{array}$ \\
\hline 1 & $42^{\circ} 43^{\prime} 47^{\prime \prime S} 71^{\circ} 45^{\prime} 24^{\prime \prime} \mathrm{W}$ & 80 & 55 \\
\hline 2 & $42^{\circ} 43^{\prime} 49^{\prime \prime S} 71^{\circ} 45^{\prime} 08^{\prime \prime} \mathrm{W}$ & 82 & 9 \\
\hline 3 & $42^{\circ} 36^{\prime} 44^{\prime \prime S} 71^{\circ} 53^{\prime} 33^{\prime \prime} \mathrm{W}$ & 0 & 18 \\
\hline 4 & $42^{\circ} 36^{\prime} 43^{\prime \prime S} 71^{\circ} 53^{\prime} 33^{\prime \prime} \mathrm{W}$ & 0 & 18 \\
\hline 5 & $42^{\circ} 43^{\prime} 44^{\prime \prime S} 71^{\circ} 45^{\prime} 22^{\prime \prime} \mathrm{W}$ & 35 & 2 \\
\hline 6 & $42^{\circ} 43^{\prime} 20^{\prime \prime S} 71^{\circ} 45^{\prime} 02^{\prime \prime} \mathrm{W}$ & 90 & 12 \\
\hline 7 & $42^{\circ} 43^{\prime} 22^{\prime \prime S} 71^{\circ} 45^{\prime} 01^{\prime \prime} \mathrm{W}$ & 55 & 0 \\
\hline 8 & $42^{\circ} 43^{\prime} 49^{\prime \prime S} 71^{\circ} 45^{\prime} 14^{\prime \prime} \mathrm{W}$ & 46 & 2 \\
\hline 9 & $42^{\circ} 43^{\prime} 47^{\prime \prime S} 71^{\circ} 45^{\prime} 16^{\prime \prime} \mathrm{W}$ & 32 & 46 \\
\hline 10 & $42^{\circ} 43^{\prime} 34^{\prime \prime S} 71^{\circ} 44^{\prime} 35^{\prime \prime} \mathrm{W}$ & 44 & 2 \\
\hline 11 & $42^{\circ} 43^{\prime} 37^{\prime \prime S} 71^{\circ} 44^{\prime} 36^{\prime \prime} \mathrm{W}$ & 9 & 2 \\
\hline 12 & $42^{\circ} 43^{\prime} 37^{\prime \prime S} 71^{\circ} 44^{\prime} 40^{\prime \prime} \mathrm{W}$ & 84 & 9 \\
\hline 13 & $42^{\circ} 43^{\prime} 40^{\prime \prime S} 71^{\circ} 44^{\prime} 42^{\prime \prime} \mathrm{W}$ & 36 & 9 \\
\hline 14 & $42^{\circ} 43^{\prime} 41^{\prime \prime S} 71^{\circ} 44^{\prime} 42^{\prime \prime} \mathrm{W}$ & 43 & 4 \\
\hline 15 & $42^{\circ} 43^{\prime} 46^{\prime \prime S} 71^{\circ} 45^{\prime} 19^{\prime \prime W}$ & 33 & 4 \\
\hline 16 & $42^{\circ} 43^{\prime} 47^{\prime \prime S} 71^{\circ} 45^{\prime} 17^{\prime \prime} \mathrm{W}$ & 24 & 0 \\
\hline 17 & $42^{\circ} 36^{\prime} 38^{\prime \prime S} 71^{\circ} 53^{\prime} 27^{\prime \prime} \mathrm{W}$ & 0 & 12 \\
\hline 18 & $42^{\circ} 43^{\prime} 48^{\prime \prime S} 71^{\circ} 45^{\prime} 011^{\prime \prime} \mathrm{W}$ & 28 & 2 \\
\hline 19 & $42^{\circ} 43^{\prime} 44^{\prime \prime S} 71^{\circ} 44^{\prime} 50^{\prime \prime} \mathrm{W}$ & 57 & 48 \\
\hline
\end{tabular}

Baar, Switzerland) to the nearest $0.1 \mathrm{~g}$. Head, pes, body and tail lengths were measured using digital Vernier callipers. Tail width was also measured, and if the animal was male, scrotal length and width were measured using callipers. Testicular volume was calculated following Celis-Diez et al. (2012) using the formula: $V=0.524 \times L \times W^{2}$, where $V=$ volume $\left(\mathrm{cm}^{3}\right), L=$ length (cm) and $W=$ width $(\mathrm{cm})$. The morphological description of pouch young was recorded.

\section{Results}

\section{Habitat surveys}

The habitat surveys confirmed Valdivian temperate forest was the key habitat of the area (for locations B and C). The habitat characteristics for each of the 19 sites are summarised in Table 2. The dominant tree species were tall evergreen beech (Nothofagus dombeyii), radal (Lomatia hirsuta) and the near threatened cordilleran cypress (Austrocedrus chilensis). Colihue native bamboo (Chusquea culeou) was observed (but dead) in nearly all sites at the time of trapping. Occasional forest sites (location B) had large Chilean myrtle (Luma apiculata), maqui (Aristotelia chilensis), tineo (Weinmannia trichosperma) and lanceleaf azara (Azara lanceolata). The understorey of most forest sites was virtually absent, with the exception of moss, and very few fern and fungi.
Most forest sites had only a few species of small shrub present. The main shrub species observed at the forest sites included Darwin's barberry (Berberis darwinii), prickly heath (Gaultheria phillyreifolia), parilla (Perilla frutescens), lanceleaf azara, young Chilean myrtle, rosea (Colletia spinosissima), mayten (Maytenus boaria), maqui and codocoipu (Spanish) (Myoschilos oblongum), with occasional specimens of vetch (Vicia magellanica) and devil's elder (Raukaua laetevirens) at some sites. Weeds were extremely rare in the forest sites.

The ex-Mermoud (location A) orchard sites were dominated by introduced stone fruit trees. These trees included apple (Malus domestica), cherry (Prunus spp.) and quince (Cydonia oblonga) and were the dominant canopy species. There was little understorey at some of these sites; however, weeds were found in abundance in more open areas at the orchard sites. The dominant shrub species in open areas at the orchard sites included Scotch broom (Cytisus scoparius) and sweet briar (Rosa rubiginosa) with a mix of short grasses and herbs.

Despite many of the trees and shrubs identified as potential sources of berries, during October, no plants had mature berries present at any of the survey sites. In November, the prickly heath and devil's elder began to fruit. The native Chilean strawberry (Fragaria chiloensis) was also beginning to fruit in some parts of the park (Gpque Cristian pers. comm.). Some immature berries were observed on Darwin's barberry in December. No hemiparasitic mistletoe (Tristerix corymbosus) was identified at any sites.

No evidence of dreys or nests was observed during the habitat surveys.

\section{Dromiciops gliroides sightings}

In the last 10 years, sightings of $D$. gliroides have been made in the PNLA (Chébez 2005), and in 2005, remains were collected from American mink (Mustela vison) scat at Lago Menéndez (42² $2^{\prime} \mathrm{S}$ ) (Fasola et al. 2008). A D. gliroides locality was provided by Martin (2010) (Table 1) for Chubut for Lago Rivadavia/Cholila; however, no further information was provided. In January 2014, a dead D. gliroides was found in Puerto Sagrario, Chubut $\left(42^{\circ}\right.$ $36^{\prime} 42.06^{\prime \prime} \mathrm{S} 71^{\circ} 53^{\prime} 28.48^{\prime \prime} \mathrm{W}$ ) (F. Castro guide PNLA pers. comm.). The intact specimen had probably just died and was found in an isolated area in the PNLA, only accessible by boat at Puerto Sagrario.

\section{Cage trapping}

Four adult D. gliroides were trapped in mid-November, 2014. Weather during the successful trapping period was fine with the temperature ranging from $6-21{ }^{\circ} \mathrm{C}$ (AccuWeather Lago Futalaufquenm 2014) with slight cloud cover. There was minimal cloud cover during the 
Table 2 Habitat survey data for each of the 19 sites surveyed for Dromiciops gliroides presence

\begin{tabular}{|c|c|c|c|c|c|c|c|c|c|c|}
\hline Site & GPS & Landscape & Vegetation type & $\begin{array}{l}\text { Dominant tree } \\
\text { species }\end{array}$ & $\begin{array}{l}\text { Dominant shrub } \\
\text { species }\end{array}$ & $\begin{array}{l}\text { Dominant ground } \\
\text { cover species }\end{array}$ & Weeds & $\begin{array}{l}\text { Tree hollows } \\
\text { (number sighted) }\end{array}$ & $\begin{array}{l}\text { Distance to } \\
\text { water }(\mathrm{m})\end{array}$ & Notes \\
\hline \multirow[t]{3}{*}{1} & \multirow{3}{*}{$\begin{array}{l}42^{\circ} 43^{\prime} 47^{\prime \prime S} 71^{\circ} \\
45^{\prime} 24^{\prime \prime} \mathrm{W}\end{array}$} & \multirow[t]{3}{*}{ Slope } & \multirow[t]{3}{*}{ Mainly native } & Nothfagus spp. & \multirow[t]{3}{*}{ Berberis darwinii } & \multirow[t]{3}{*}{ Moss (limited) } & \multirow[t]{3}{*}{ No } & \multirow[t]{3}{*}{89} & & \multirow{3}{*}{$\begin{array}{l}\text { Dead Chusquea culeou (some } \\
\text { fallen) }\end{array}$} \\
\hline & & & & Lomatia hirsuta & & & & & & \\
\hline & & & & Chusquea culeou & & & & & & \\
\hline \multirow[t]{4}{*}{2} & \multirow{4}{*}{$\begin{array}{l}42^{\circ} 43^{\prime} 49^{\prime \prime} \mathrm{S} 71^{\circ} \\
45^{\prime} 08^{\prime \prime} \mathrm{W}\end{array}$} & \multirow[t]{4}{*}{ Flat } & \multirow[t]{4}{*}{ Mainly native } & Nothfagus spp. & Berberis darwinii & \multirow[t]{4}{*}{ Moss } & \multirow[t]{4}{*}{ No } & \multirow[t]{4}{*}{3} & \multirow{4}{*}{$\begin{array}{l}7 \mathrm{~m} \text { to Rio } \\
\text { Menendez }\end{array}$} & \\
\hline & & & & Lomatia hirsuta & Gaultheria mucronata & & & & & \\
\hline & & & & Chusquea culeou & Myoschilos oblongum & & & & & \\
\hline & & & & $\begin{array}{l}\text { Austrocedrus } \\
\text { chilensis }\end{array}$ & Colletia spinosissima & & & & & \\
\hline \multirow[t]{2}{*}{3} & \multirow{2}{*}{$\begin{array}{l}42^{\circ} 36^{\prime} 44^{\prime \prime S} 71^{\circ} \\
53^{\prime \prime} 33^{\prime \prime} \mathrm{W}\end{array}$} & \multirow[t]{2}{*}{ Flat } & \multirow[t]{2}{*}{ Mainly native } & Nothfagus spp. & \multirow[t]{2}{*}{ Azara lanceolata } & Fern & \multirow[t]{2}{*}{ No } & \multirow[t]{2}{*}{7} & \multirow[t]{2}{*}{$50 \mathrm{~m}$} & Chusquea culeou; Vine Mitraria \\
\hline & & & & Chusquea culeou & & Moss & & & & Lycalopex culpaeus scats \\
\hline \multirow[t]{2}{*}{4} & \multirow{2}{*}{$\begin{array}{l}42^{\circ} 36^{\prime} 43^{\prime \prime S} 71^{\circ} \\
53^{\prime} 33^{\prime \prime} \mathrm{W}\end{array}$} & \multirow[t]{2}{*}{ Flat } & \multirow[t]{2}{*}{ Mainly native } & Chusquea culeou & Azara lanceolata & Moss & No & 0 & $40 \mathrm{~m}$ & Lycalopex culpaeus scats \\
\hline & & & & & Mitraria coccinea & Ferns (occasional) & & & & \\
\hline 5 & $42^{\circ} 43^{\prime} 44^{\prime \prime} S 71^{\circ}$ & Flat & Mainly native with & Nothfagus spp. & Lomatia hirsuta & Moss & Yes & 29 & $250 \mathrm{~m}$ & \\
\hline & & & some weeds & Lomatia hirsuta & Berberis darwinii & & $\begin{array}{l}\text { Rosa } \\
\text { rubigin osa }\end{array}$ & & & \\
\hline & & & & Chusquea culeou & Maytenus boaria & & Ribes spp. & & & \\
\hline & & & & Luma apiculata & & & & & & \\
\hline 6 & $42^{\circ} 43^{\prime} 20^{\prime \prime S} 71^{\circ}$ & Slope & Mainly weeds with & Prunus spp. & Rosa rubiginosa & Fern & Yes & 0 & $50 \mathrm{~m}$ & Bottom of ridge \\
\hline & & & & Azara lanceolata & Berberis darwinii & & Rosa & & & Evidence of pig rooting \\
\hline & & & & Luma apiculata & & & & & & Lepus europaeus scats \\
\hline 7 & $42^{\circ} 43^{\prime} 22^{\prime \prime S} 71^{\circ}$ & Flat & Mainly weeds with & Malus domestica & Rosa rubiginosa & Grasses & Yes & 0 & $200 \mathrm{~m}$ & Malus domestica flowering \\
\hline & & & & Cydonia oblonga & Malus domestica & Fragaria chiloensis & $\begin{array}{l}\text { Rosa } \\
\text { rubiginosa }\end{array}$ & & & \\
\hline & & & & & Chusquea culeou & & $\begin{array}{l}\text { Cytisus } \\
\text { scoparius }\end{array}$ & & & Evidence of pig rooting \\
\hline & & & & & & & $\begin{array}{l}\text { Trifolium } \\
\text { sp. }\end{array}$ & & & Lepus europaeus scats \\
\hline 8 & $42^{\circ} 43^{\prime} 49^{\prime \prime} \mathrm{S} 71^{\circ}$ & Slope & Mainly native & Nothfagus spp. & Colletia spinosissima & Moss & No & 69 & $10 \mathrm{~m}$ & \\
\hline & & & & Lomatia hirsuta & Berberis darwinii & & & & & \\
\hline & & & & Chusquea culeou & Aristotelia chilensis & & & & & \\
\hline & & & & $\begin{array}{l}\text { Austrocedrus } \\
\text { chilensis }\end{array}$ & & & & & & \\
\hline 9 & $\begin{array}{l}42^{\circ} 43^{\prime} 47^{\prime \prime} 571^{\circ} \\
45^{\prime \prime} 6^{\prime \prime} \mathrm{W}\end{array}$ & Slope & Mainly native & Nothfagus spp. & Gaultheria mucronata & Moss & No & 10 & $10 \mathrm{~m}$ & Some Chusquea culeou still green, \\
\hline & & & & Lomatia hirsuta & Myoschilos oblongum & & & & & \\
\hline
\end{tabular}


Table 2 Habitat survey data for each of the 19 sites surveyed for Dromiciops gliroides presence (Continued)

\begin{tabular}{|c|c|c|c|c|c|c|c|c|c|c|}
\hline & & & & Chusquea culeou & Colletia spinosissima & & & & & \\
\hline & & & & $\begin{array}{l}\text { Austrocedrus } \\
\text { chilensis }\end{array}$ & Vicia magellanica & & & & & \\
\hline & & & & Luma apiculata & & & & & & \\
\hline & & & & Aristotelia chilensis & & & & & & \\
\hline \multirow[t]{6}{*}{10} & \multirow{6}{*}{$\begin{array}{l}42^{\circ} 43^{\prime} 34^{\prime \prime} \mathrm{S} 71^{\circ} \\
44^{\prime} 35^{\prime \prime} \mathrm{W}\end{array}$} & \multirow{6}{*}{$\begin{array}{l}\text { Top of } \\
\text { ridge }\end{array}$} & \multirow[t]{6}{*}{ Mainly native } & Nothfagus spp. & \multicolumn{2}{|l|}{ Raukaua laetevirens } & Yes & \multirow[t]{6}{*}{0} & \multirow[t]{6}{*}{$100 \mathrm{~m}$} & \multirow{3}{*}{$\begin{array}{l}\text { Fresh pig scat, bird bones, bird } \\
\text { scats, possible mink poo }\end{array}$} \\
\hline & & & & Lomatia hirsuta & Berberis darwinii & & \multirow{5}{*}{$\begin{array}{l}\text { Rosa } \\
\text { rubiginosa }\end{array}$} & & & \\
\hline & & & & Chusquea culeou & Aristotelia chilensis & & & & & \\
\hline & & & & $\begin{array}{l}\text { Austrocedrus } \\
\text { chilensis }\end{array}$ & & & & & & $\begin{array}{l}\text { Lycalopex culpaeus sighted near } \\
\text { this site } 42^{\circ} 43^{\prime} 34^{\prime \prime S} \mathrm{~S} 71^{\circ} 44^{\prime} 33^{\prime \prime} \mathrm{W}\end{array}$ \\
\hline & & & & Luma apiculata & & & & & & \\
\hline & & & & Aristotelia chilensis & & & & & & \\
\hline \multirow[t]{4}{*}{11} & \multirow{4}{*}{$\begin{array}{l}42^{\circ} 43^{\prime} 37^{\prime \prime S} 71^{\circ} \\
44^{\prime} 36^{\prime \prime} \mathrm{W}\end{array}$} & \multirow[t]{4}{*}{ Flat } & \multirow[t]{4}{*}{ Mainly native } & \multirow[t]{2}{*}{ Lomatia hirsuta } & Vicia magellanica & & \multirow[t]{2}{*}{ Yes } & \multirow[t]{4}{*}{0} & \multirow[t]{4}{*}{$20 \mathrm{~m}$} & \multirow[t]{4}{*}{ Dead trees } \\
\hline & & & & & Berberis darwinii & & & & & \\
\hline & & & & \multirow{2}{*}{$\begin{array}{l}\text { Austrocedrus } \\
\text { chilensis }\end{array}$} & Gaultheria mucronata & & \multirow{2}{*}{$\begin{array}{l}\text { Rosa } \\
\text { rubiginosa }\end{array}$} & & & \\
\hline & & & & & Myoschilos oblongum & & & & & \\
\hline \multirow[t]{3}{*}{12} & \multirow{3}{*}{$\begin{array}{l}42^{\circ} 43^{\prime} 37^{\prime \prime S} 71^{\circ} \\
44^{\prime} 40^{\prime \prime} \mathrm{W}\end{array}$} & \multirow[t]{3}{*}{ Flat } & \multirow[t]{3}{*}{ Mainly native } & Nothfagus spp. & Aristotelia chilensis & \multirow[t]{10}{*}{ Bracken fern } & Yes & \multirow[t]{3}{*}{0} & \multirow[t]{3}{*}{$30 \mathrm{~m}$} & \multirow{3}{*}{$\begin{array}{l}\text { Fresh pig scats; road through } \\
\text { transect }\end{array}$} \\
\hline & & & & Lomatia hirsuta & Vicia magellanica & & Rosa & & & \\
\hline & & & & $\begin{array}{l}\text { Austrocedrus } \\
\text { chilensis }\end{array}$ & & & & & & \\
\hline 13 & $42^{\circ} 43^{\prime} 40^{\prime \prime} \mathrm{S} 71^{\circ}$ & Flat & mainly native & Nothfagus spp. & Gaultheria mucronata & & No & 0 & $30 \mathrm{~m}$ & Road through transect \\
\hline & $44^{\prime} 42^{\prime \prime W}$ & & & Lomatia hirsuta & Berberis darwinii & & & & & \\
\hline & & & & Chusquea culeou & Raukaua laetevirens & & & & & \\
\hline & & & & $\begin{array}{l}\text { Austrocedrus } \\
\text { chilensis }\end{array}$ & & & & & & \\
\hline & & & & Luma apiculata & & & & & & \\
\hline 14 & $42^{\circ} 43^{\prime} 41^{\prime \prime S} 71^{\circ}$ & Slight & Mainly native & Lomatia hirsuta & Aristotelia chilensis & & No & 0 & $60 \mathrm{~m}$ & Fresh pig scats \\
\hline & & & & Chusquea culeou & Maytenus boaria & & & & & \\
\hline 15 & $42^{\circ} 43^{\prime} 46^{\prime \prime S} 71^{\circ}$ & Ridge & Mainly native & Nothfagus spp. & Berberis darwinii & Moss & No & 15 & $20 \mathrm{~m}$ & Some evidence of disturbance \\
\hline & & & & Lomatia hirsuta & Maytenus boaria & Austrocedrus & & & & \\
\hline & & & & Chusquea culeou & & chilensis fungl & & & & \\
\hline & & & & $\begin{array}{l}\text { Austrocedrus } \\
\text { chilensis }\end{array}$ & & Brain fungi & & & & \\
\hline & & & & Luma apiculata & & & & & & \\
\hline & & & & Aristotelia chilensis & & & & & & \\
\hline 16 & $\begin{array}{l}42^{\circ} 43^{\prime} 47^{\prime \prime S} \mathrm{~S} 71^{\circ} \\
45^{\prime} 17^{\prime \prime} \mathrm{W}\end{array}$ & flat & Mainly native & Nothfagus spp. & Colletia spinosissima & Moss & No & 19 & & $\begin{array}{l}\text { Bird print, mink print at the water } \\
\text { edge, sand, beach }\end{array}$ \\
\hline
\end{tabular}


Table 2 Habitat survey data for each of the 19 sites surveyed for Dromiciops gliroides presence (Continued)

\begin{tabular}{|c|c|c|c|c|c|c|c|c|c|c|}
\hline & & & & Chusquea culeou & Myoschilos oblongum & & & & & \\
\hline & & & & $\begin{array}{l}\text { Austrocedrus } \\
\text { chilensis }\end{array}$ & & & & & & \\
\hline & & & & Luma apiculata & & & & & & \\
\hline & & & & $\begin{array}{l}\text { Weinmannia } \\
\text { trichosperma }\end{array}$ & Gaultheria mucronata & & & & & \\
\hline \multirow[t]{6}{*}{17} & \multirow{6}{*}{$\begin{array}{l}42^{\circ} 36^{\prime} 38^{\prime \prime S} 71^{\circ} \\
53^{\prime} 27^{\prime \prime} \mathrm{W}\end{array}$} & \multirow{6}{*}{$\begin{array}{l}\text { Slight } \\
\text { slope }\end{array}$} & \multirow[t]{6}{*}{ Mainly native } & Nothfagus spp. & Azara lanceolata & Moss & \multirow[t]{6}{*}{ No } & \multirow[t]{6}{*}{11} & \multirow[t]{6}{*}{$60 \mathrm{~m}$} & \multirow{6}{*}{$\begin{array}{l}\text { Some green Chusquea culeou, } \\
\text { walkway construction materials } \\
\text { in transect }\end{array}$} \\
\hline & & & & Lomatia hirsuta & Raukaua laetevirens & Ferns $\times 2$ & & & & \\
\hline & & & & Chusquea culeou & & & & & & \\
\hline & & & & $\begin{array}{l}\text { Austrocedrus } \\
\text { chilensis }\end{array}$ & & & & & & \\
\hline & & & & $\begin{array}{l}\text { Fitzroya } \\
\text { cupressoides }\end{array}$ & & & & & & \\
\hline & & & & $\begin{array}{l}\text { Weinmannia } \\
\text { trichosperma }\end{array}$ & & & & & & \\
\hline \multirow[t]{6}{*}{18} & \multirow{6}{*}{$\begin{array}{l}42^{\circ} 43^{\prime} 48^{\prime \prime S} 71^{\circ} \\
45^{\circ} 01^{\prime \prime} \mathrm{W}\end{array}$} & \multirow[t]{6}{*}{ Slope } & \multirow[t]{6}{*}{ Mainly native } & Nothfagus spp. & Myoschilos oblongum & Moss & \multirow[t]{6}{*}{ No } & \multirow[t]{6}{*}{25} & \multirow[t]{6}{*}{$10 \mathrm{~m}$} & \\
\hline & & & & Lomatia hirsuta & Gaultheria mucronata & & & & & \\
\hline & & & & Chusquea culeou & Berberis darwinii & & & & & \\
\hline & & & & $\begin{array}{l}\text { Austrocedrus } \\
\text { chilensis }\end{array}$ & Maytenus boaria & & & & & \\
\hline & & & & Luma apiculata & Raukaua laetevirens & & & & & \\
\hline & & & & Aristotelia chilensis & & & & & & \\
\hline \multirow[t]{7}{*}{19} & \multirow{7}{*}{$\begin{array}{l}42^{\circ} 43^{\prime} 44^{\prime \prime S} 71^{\circ} \\
44^{\prime} 50^{\prime \prime} \mathrm{W}\end{array}$} & \multirow[t]{7}{*}{ Slope } & \multirow[t]{7}{*}{ Mainly native } & Nothfagus spp. & Myoschilos oblongum & Moss & \multirow[t]{7}{*}{ No } & \multirow[t]{7}{*}{25} & \multirow[t]{7}{*}{$10 \mathrm{~m}$} & \multirow[t]{7}{*}{ Lycalopex culpaeus scats } \\
\hline & & & & Austrocedrus & Gaultheria mucronata & & & & & \\
\hline & & & & & Berberis darwinii & & & & & \\
\hline & & & & Lomatia hirsuta & Maytenus boaria & & & & & \\
\hline & & & & Chusquea culeou & Vicia magellanica & & & & & \\
\hline & & & & $\begin{array}{l}\text { Austrocedrus } \\
\text { chilensis }\end{array}$ & & & & & & \\
\hline & & & & Aristotelia chilensis & & & & & & \\
\hline
\end{tabular}


October and December surveying periods, when no animals were trapped.

All $D$. gliroides were captured in forest habitats at three different sites (1, 9 and 19). No other species were trapped. Both males (2) and females (2) were captured. The cage trap that trapped male 1 (site 1) had been placed on a horizontal dead branch nearby to some native bamboo and radal, approximately $1 \mathrm{~m}$ from the ground. Male 2 and female 1 were captured at the same site (site 19), using the same trap, with the female being captured 2 days after the male. This trap was placed on the horizontal branch of a large cypress, near a large beech, approximately $2 \mathrm{~m}$ from the ground. The trap entrance was facing the cypress tree trunk. Female 2 was captured at site 9 , on the same day as female 1 . The trap had been placed on a fallen branch approximately $0.5 \mathrm{~m}$ from the ground.

All three baits were successful in attracting $D$. gliroides into the cage traps. Both males consumed all the bait in the traps, banana (male 1) and apple (male 2). In comparison, both females appeared to have not eaten any of the bait left in the cage traps (apple (female 1) and bait ball (female 2)).

\section{Infrared camera trapping}

Camera trap 4 (site 19) recorded D. gliroides on five nights in mid to late November. The videos showed the animal/s moving along branches of a cypress tree. Cameras 1 (two nights) and 5 (two nights) recorded foxes (Lycalopex culpaeus). The videos showed a single fox walking past the camera, and in some videos sniffing around the areas.

\section{Morphological data}

Both adult males (2) and females (2) were captured. All animals were regarded as healthy, having no obvious signs of ill-health and were within normal weight range for the species. The coats of all animals appeared in good condition, even though female 1 had fleas (unidentified species). Table 3 summarises the morphological measurements taken for each adult $D$. gliroides.

The scrotum of the males was examined and measured. The left side of the scrotal sac of male 1 was blue grey and the right side pink; however, this was the opposite for male 2 . The scrotal sac width of male 1 was $14.65 \mathrm{~mm}$ and male $212.48 \mathrm{~mm}$. The length of the scrotal sac was 7.15 and $8.35 \mathrm{~mm}$ for Male 1 and 2, respectively. Male 1 had a scrotal volume of $0.80 \mathrm{~cm}^{3}$ and male 2 a scrotal volume of $0.68 \mathrm{~cm}^{3}$.

Both females captured had pouch young in an enclosed pouch. Female 1 had at least three and female 2 at least two young. All pouch young lacked fur, had no pigmentation and were firmly attached to teats deep in the pouch. To minimise mis-mothering, only one pouch
Table 3 Morphological data for adult male and female Dromiciops gliroides captured

\begin{tabular}{lllll}
\hline Animal identification & Male 1 & Male 2 & Female 1 & Female 2 \\
\hline Weight $(\mathrm{g})$ & 24.5 & 27 & 34.5 & 26.5 \\
Total length $(\mathrm{mm})$ & 191.65 & 217.99 & 205.61 & 204.54 \\
Length snout to rump $(\mathrm{mm})$ & 93.99 & 102.34 & 105.55 & 103.11 \\
Head length $(\mathrm{mm})$ & 31.71 & 29.71 & 30.66 & 32.28 \\
Pes length $(\mathrm{mm})$ & 17.17 & 14.44 & 15.16 & 17.56 \\
Ear length $(\mathrm{mm})$ & 6.99 & 12.93 & 7 & 10.76 \\
Tail length $(\mathrm{mm})$ & 97.66 & 115.65 & 100.06 & 101.43 \\
Tail diameter $(\mathrm{mm})$ & 5.55 & 6.16 & 5.93 & 8.98 \\
\hline
\end{tabular}

young from each litter was measured. The pouch young of female 1 had a crown to rump length of $13.33 \mathrm{~mm}$, and the crown to rump length of the pouch young of female 2 was $20.84 \mathrm{~mm}$. Neither of the two pouch young investigated in detail had their eyes open; however, toes and distal claws were visible on the hind feet, tails were detached, and the ears were starting to form and detach. The genital tubercle was visible only in the pouch young from female 2 .

\section{Further observations}

Sites 10, 12 and 14 had evidence of pig activity, specifically fresh pig scats. Open areas near to sites 6 and 7 (old orchards) had large areas where pig rooting and foraging was evident. European hare (Lepus europaeus) scats were present too.

Consistent with the camera trap data, foxes (Lycalopex culpaeus) were opportunistically observed during the day, one near the Pasarella (Río Arrayanes footbridge) $\left(42^{\circ} 43^{\prime} 34^{\prime \prime} \mathrm{S} 71^{\circ} 44^{\prime} 33^{\prime \prime} \mathrm{W}\right)$ and another near site 1 and Puerto Chucao. Fox scats were observed at sites 3, 4 and 19, and fox prints were observed at several locations throughout the survey sites including along the track from the Pasarella to Puerto Chucao.

American mink were observed during the day opportunistically whilst walking between different sites $\left(42^{\circ} 43^{\prime}\right.$ $50^{\prime \prime} \mathrm{S} 71^{\circ} 45^{\prime} 08^{\prime \prime} \mathrm{W}$ and $\left.42^{\circ} 43^{\prime} 35^{\prime \prime} \mathrm{S} 71^{\circ} 44^{\prime} 47^{\prime \prime} \mathrm{W}\right)$. Mink tracks were also observed along the tracks in close proximity to the water.

Domestic animals were present in the park. Within the survey area, we observed one domestic cat (Felis catus) at Puerto Chucao, in close proximity to site 1 . Another adult cat was observed in the carpark adjacent to the Pasarella, and three additional domestic cats were observed in other areas in the park. One large domestic dog (Canis familiaris) was also observed during site surveys.

Local residents and National Park staff reported high numbers of owls during the period when the bamboo was flowering and producing seeds. These reports were 
supported by the very large numbers of owl pellets found throughout the survey sites.

\section{Discussion}

Our study has confirmed the presence of the near threatened arboreal marsupial $D$. gliroides at a latitude as south as $42^{\circ} 43^{\prime} \mathrm{S}$ in the PNLA, Chubut province, Argentina. D. gliroides is more commonly known to inhabit the temperate forests of Río Negro and Neuquén (Birney et al. 1996) and in the adjacent areas of Chile and the Island of Chiloé (Patterson et al. 1990). However, Heinonen Fortabat and Chébez (1997) first suggested the possibility that $D$. gliroides may be present further south in the province of Chubut and included it in the species list for the PNLA. Hence, prior to this study, the distribution of $D$. gliroides in Argentina extended only to $42^{\circ} 32^{\prime} 58^{\prime \prime} \mathrm{S} 71^{\circ} 37^{\prime} 56^{\prime \prime} \mathrm{W}$ for Chiloé /Lago Rivadavia in Northern Chubut, Argentina (Martin 2010); however, no other information is given about this finding.

Both male and female $D$. gliroides were caught during trapping, and the females had pouch young present. We estimate based on crown rump length measurements (Frankham and Temple-Smith 2012) that the pouch young were between 15 and 30 days old. The presumed ages of the pouch young suggest birth occurred in early October and mating in early spring or late winter, and is similar to other records of pouch young in other parts of Argentina (e.g. Pearson 1983; Gurovich unpublished data), Chile (e.g. Muñoz-Pedreros et al. 2005; Frankham and Temple-Smith 2012) and the island of Chiloé (CelisDiez et al. 2012).

Males caught in November 2014 were slightly larger than adults caught in April $(19.5 \pm 0.5 \mathrm{~g})$ by Pearson (1983) and slightly smaller than those caught in summer $(28.9 \pm 1.4$ g; January-March 2000 and 2001) in Argentina (Rodríguez-Cabal et al. 2008). Females were, on average, heavier and slightly longer than the males and similar to observations made by Rodríguez-Cabal et al. (2008) in Río Negro, Argentina. The tail diameter of the individuals caught in this study were slightly smaller for both males and females compared to those caught in summer $(7-9 \mathrm{~mm})$ and winter $(10-13 \mathrm{~mm})$ in Chile (Kelt and Martínez 1989).

Based on the health condition of the males captured in this study, we suggest that it is unlikely that $D$. gliroides are semelparous and do not experience $100 \%$ male mortality following an intense mating period as seen in some Australian dasyurid marsupials (e.g. Bradley 1997), and at least five species of South American Didelphids (see Lopes and Leiner 2014). However, in order to confirm this, further studies are required.

A significant number of trap nights were required to trap D. gliroides within the PNLA, with the success rate $<1 \%$. Celis-Diez et al. (2012) had a low capture rate in spring (2/ha) compared to summer (20/ha) in Chiloé. However, these two studies differ from other studies where $D$. gliroides appears to be easily captured (using the same bait) such as Rodríguez-Cabal and Branch (2011), in which they stated they could capture D. gliroides within four nights ( $99.6 \%$ success rate) if it was present. However, given the low capture rates of this study, the statements by Rodríguez-Cabal and Branch (2011) is likely not the case at all sites. The difference in habitat was evident in our study sites compared to the study sites of Rodríguez-Cabal and Branch (2011), whereby bamboo was abundant and there was mistletoe present. In our study site, the bamboo had flowered and seeded between 2012 and 2014 and there was only the occasional exceptionally rare bamboo seedling observed. Mistletoe was also lacking at all our study sites; however, it has previously been noted not to preclude $D$. gliroides from a site (Martin 2010).

As this is the first confirmation that D. gliroides are present within the PNLA, their diet in the area and if they even consume fruits remain unknown. Given the lack of fruit at the time we captured $D$. gliroides, it suggests their diet was likely to be high in insects. A diet rich in insects during the time young are reared is presumably beneficial due to higher protein levels. This is similar to the mountain pygmy-possum (Burramys par$v u s$ ) whose diet generally follows seasonal availability of arthropods (Smith and Broome 1992). However, female pygmy-possums consume more arthropods than males most likely due to their higher protein requirement for reproduction and to provide sustenance for their young, allowing them to grow to an adequate size in preparation for winter hibernation (Smith and Broome 1992). Similarly, the opossum (Caluromys philander) increases consumption of insects to coincide with late lactation Tyndale-Biscoe (2005), and the red-tailed phascogale (Phascogale calura) increases consumption of vertebrates as opposed to eating insects during the breeding season (Stannard et al. 2010). However, further diet studies are needed to support this observation.

All successful trapping (cage and cameras) occurred in relatively undisturbed Nothofagus forest in small branches (live or dead) $0.5-2 \mathrm{~m}$ from the ground, and between 40 and $80 \mathrm{~m}$ from a water source. D. gliroides was the most abundant (and the only) species of small mammal caught in the understorey stratum in this study similar to what was reported by Rodríguez-Cabal et al. (2008) who also found D. gliroides as the most abundant small mammal in the understorey, but not the only small mammal (20\% in 2000 and $17 \%$ in 2001 all captures).

During late 2013 to mid-2014, there was an increase in rodent numbers (up to $600 \%$ ) in the PNLA related to the bamboo flowering and seeding event. Trapping efforts by National Park Rangers and 
inhabitants of the PNLA reported trapping hundreds of rodents during 2013 and up to 1000 rodents per hectare during November 2013 (Gpque Sanchez pers. comm.). Our trapping occurred post bamboo flowering and seeding, and post rodent irruption. During the irruption, vast numbers of rodents needed to seek food and many trees and shrubs are likely to have suffered. Given the vast numbers of rodents and their appetites, it is likely that it impacted on the ability of the understorey and mid-storey plants to re-establish quickly after the bamboo flowered, seeded and died. The decrease in understorey and mid-storey levels may have also impacted on the ability of $D$. gliroides to move between different areas and to find suitable food and shelter. During the rodent irruption, numbers of predators increased (e.g. owls) and likely impacted directly on D. gliroides; however, further studies to understand the ecosystem dynamics as these times are required.

Threats to D. gliroides and its habitat are not unique to the PNLA. These include natural and introduced predators and human impacts from farming and tourism. Introduced predators such as mink and domestic cats and dogs are likely to impact directly on $D$. gliroides. American mink was introduced to Patagonia in the early to mid-twentieth century for fur farming (Lizarralde and Escobar 2000). They are present in the PNLA and are known to prey on D. gliroides (Fasola et al. 2008), and they were observed throughout the study area during surveys. Domestic cats were observed in the PNLA during the trapping period. The threats posed by cats to native wildlife including native marsupials have been documented at length in various habitats in Australia (e.g. Abbott 2002) and near Llao Llao in Bariloche, Prov. De Río Negro domestic cats are known to hunt and kill D. gliroides (G. Amico pers. comm.), and has been detected in Parque Municipal Llao Llao by Di Virgilio et al. (2014).

Dromiciops gliroides have been shown to make nests out of bamboo leaves (e.g. Hershkovitz 1999). In this study, we did not observe any dreys and there was no bamboo suitable for building nests. Field observations have however suggested that $D$. gliroides nests in tree hollows (Franco et al. 2011) similar to Australasian marsupials (Geiser 1994; Kerle et al. 2013), and that they use introduced nest boxes (Franco et al. 2011; Celis-Diez et al. 2012). A lack of mature trees with hollows in the PNLA would therefore reduce potential nesting sites for $D$. gliroides and hence reduce the habitat available to them. Introduced livestock also known to browse on Nothofagus and alerce saplings in the PNLA (Vila and Borelli 2011) would indirectly impact on D. gliroides by reducing the number of mature trees in the longer-term and hence nesting sites.
Finally, tourism although not likely to directly impact on $D$. gliroides is likely to lead to impacts on vegetation through soil impaction from walking off paths and possibly from the transmission of Phytophthora spp. (an organism that causes root rot) spread by human activity, causing mortality to cypress trees (Filip and Rosso 1999). It is possible the high volume of predators and threats from domestic animals and tourism contributed to the low capture rate of $D$. gliroides during the study.

\section{Conclusions}

This study presents unequivocal evidence of the presence of $D$. gliroides in the PNLA and confirms its presence in the province of Chubut, Argentina. Further studies are required to elucidate the diet of $D$. gliroides within the PNLA and to uncover further aspects of its biology, especially those relating to the impacts of the flowering and seeding of the native bamboo and the resultant rodent irruption.

\section{Ethical approval}

All procedures performed in this study involving animals were in accordance with the ethical standards of the institution or practice at which the studies were conducted. Permission to trap and handle D. gliroides was issued by Parques Nacionales (Argentina) (Permit Number: DPR1344) and agreed with the ethical principles on animal research and handling rare animals in Patagonia according to Administración de Parques Nacionales (Argentina). Ethics approval was also granted by Western Sydney University (Australia) animal ethics committee, having met the Australian Code of Practice for the Care and Use of Animals for Scientific Purposes. This article does not contain any studies with human participants performed by any of the authors.

\section{Competing interests}

The authors declare that they have no competing interests.

\section{Authors' contributions}

YG conceived and coordinated the study. All authors worked within the acquisition of the permits and logistics of the study, designed and participated in the fieldwork, collected data, analysed the data, interpreted the data and results, and drafted the manuscript. All authors read and approved the final manuscript.

\section{Acknowledgements}

We thank Dr. Guillermo Amico and Lic Agustina Balazote for support and discussion as well as Dr. Richard Sage for help and encouragement. We thank the volunteers that helped with trapping and logistics - Park Rangers Gpque. Gustavo Sanchez (Sec. Lago Verde), Gpque. Christian Vellido (Sec. Arrayanes) and Gpque. Gustavo Paramosz (Sec. Puerto Chucao), and volunteer trappers (JS. Adams, L. and F. Gurovich, S. Gurovich and CJ. Henderson). We thank Mr. De Beranardi of Cleona for transport via boat to and from Puerto Sagrario. Also help from Lic. Hernan Pastore (Delegación Regional Patagonia), Gpque Martín Izquierdo (Conservación Parque Nacional Los Alerces) and Intendente de Parque Nacional Los Alerces Daniel Crosta. 


\section{Funding}

Funding was awarded to Old from the Water and Wildlife Ecology, School of Science and Health, WSU for equipment and travel. Funding for field work was awarded to Gurovich from CONICET.

\section{Author details \\ ${ }^{1}$ National Scientific and Technical Research Council (CONICET) and CIEMEP, Laboratory of Investigative Evolution and Biodiversity (LIEB), National University of Patagonia, San Juan Bosco, Roca 780, 9200 Esquel, Chubut, Argentina. ${ }^{2}$ UNSW, Sydney, Australia. ${ }^{3}$ Water and Wildlife Ecology, School of Science and Health, Western Sydney University, Penrith, NSW 2751, Australia. ${ }^{4}$ Present address: School of Biological Sciences and Charles Perkins Centre, University of Sydney, Sydney, NSW 2006, Australia.}

Received: 20 June 2015 Accepted: 6 October 2015 Published online: 15 October 2015

\section{References}

Abbott I (2002) Origin and spread of the cat, Felis catus, on mainland Australia, with a discussion of the magnitude of its early impact on native fauna. Wildl Res 29:51-74

AccuWeather Lago Futalaufquenm Argentina (2014) http://www.accuweathercom/ en/ar/lago-futalaufquen/8227/month/8227?monyr=11/01/2014 Viewed 10.12.14

Amico GC, Aizen MA (2000) Mistletoe seed dispersal by a marsupial. Nature 408:929-930

Amico GC, Rodríguez-Cabal MA, Aizen MA (2009) The potential key seeddispersing role of the arboreal marsupial Dromiciops gliroides. Acta Oecol 35:8-13

APN (1997) Plan preliminar de manejo del Parque Nacional Los Alerces. Administracion de Parques Nacionales, Bariloche, Argentina, p 87

Birney EC, Sikes RS, Monjeau JA, Guthman N, Phillips CJ (1996) Comments on Patagonian marsupials from Argentina. In: Genoways HH, Baker RJ (eds) Contributions in Mammalogy: A Memorial Volume Honoring Dr. J. Knox Jones. Jr. Museum of Texas Tech University, Lubbock, Texas, USA, pp 149-154

Bisheimer MV, Fernández EM (2009) Árboles de los parques nacionales del sur: árboles autóctonos característicos de los bosques andino-patagónicos de la Argentina. Tercera Edición. Autores Editores, Buenos Aires, Argentina

Bradley AJ (1997) Reproduction and life history in the red-tailed phascogale, Phascogale calura (Marsupialia: Dasyuridae): the adaptive stress senescence hypothesis. J Zool 241:739-755. doi:10.1016/0016-6480(87) 90208

Cabrera LA (1971) Fitogeografía de la República Argentina. Bol Soc Argent Bot $1: 1-42$

Celis-Diez JL, Hetz J, Marín PA, Fuster G, Necochea P, Vásquez RA, Jaksic FM, Armesto JJ (2012) Population abundance, natural history, and habitat use by the arboreal marsupial Dromiciops gliroides in rural Chiloé Island, Chile. J Mammal 93:134-148

Chébez JC (2005) Guía de las Reservas Naturales de la Argentina. Ed. Albatros, Patagonia Austral. Buenos Aires

Di Virgilio A, Amico GC, Morales JM (2014) Behavioral traits of the arboreal marsupial Dromiciops gliroides during Tristerix corymbosus fruiting season. J Mammal 95:1189-1198

Diez M, Teta P (2008) Dromiciops gliroides. In: IUCN 2013. IUCN Red List of Threatened Species. Version 2013.1. www.iucnredlist.org

Dimitri MJ (1977) Pequeña flora ilustrada de los parques nacionales andinopatagónicos. Publicación Técnica $N^{\circ}$ 46. Instituto Nacional de Tecnología Agropecuaria (INTA), Buenos Aires, Argentina

Fasola L, Cassini G, Cassini M, Sage R (2008) Restos de Dromiciops gliroides en el Parque Nacional Los Alerces, provincia de Chubut, Argentina. 3 pp. In: Administración de Parques Nacionales. Sistema de Información de Biodiversidad. Proyecto de Conservación de la Biodiversidad - Donación GEF-BIRF TF 028372-AR. www.parquesnacionales.gov.ar

Filip GM, Rosso PH (1999) Cypress mortality (mal del ciprés) in the Patagonian Andes: comparison with similar forest diseases and declines in North America. Eur J For Path 29:89-96

Fontúrbel FE, Silva-Rodríguez EA, Cárdenas NH, Jiménez JE (2010) Spatial ecology of monito del monte (Dromiciops gliroides) in a fragmented landscape of southern Chile. Mammal Biol 75:1-9

Franco M, Quijano A, Soto-Gamboa M (2011) Communal nesting, activity patterns, and population characteristics in the near-threatened monito del monte, Dromiciops gliroides. J Mammal 92:994-1004
Frankham GJ, Temple-Smith PD (2012) Absence of mammary development in male Dromiciops gliroides: another link to the Australian marsupial fauna. J Mammal 93:572-578

Geiser, F (1994) Hibernation and daily torpor in marsupials: a review. Aust J Zool 42: 1-16.

Heinonen Fortabat SH, Chébez JC (1997) Los mamíferos de los parques nacionales de la Argentina, Monografla especial. Literature of Latin America 14:1-76

Hershkovitz P (1999) Dromiciops gliroides Thomas, 1894, last of the Microbiotheria (Marsupialia), with a review of the family Microbiotheriidae. Fieldiana Zool 93:1-60

Hoermann I (2013) Flora Nativa Guia de Campo Parques Nacional Los Alerces, Trevelin y Esquel. Dunken, Buenos Aires

Jiménez J, Rageot R (1979) Notas sobre la biología del "monito del monte", Dromiciops australis Philippi 1893. An Mus Hist Nat Valpso 12:83-88

Kelt DA, Martínez DR (1989) Notes on the distribution and ecology of two marsupials endemic to the Valdivian forests of Southern South America. J Mammal 70:220-224

Kerle JA, Kimmorley C, Old JM (2013) An inland population of the common ringtail possum (Pseudocheirus peregrinus) at Black Rock Ridge, New South Wales: a preliminary ecological assessment. Aust Mammal 35:236-240

Kirsch JAW, Lapointe FJ, Springer MS (1997) DNA-hybridization studies of marsupials and their implications for metatherian classification. Aust J Zool 45:211-280

Lizarralde MS, Escobar JM (2000) Exotic mammals in Tierra del Fuego. Ciencia Hoy 10:52-63

Lobos G, Charrier A, Carrasco G, Palma RE (2005) Presence of Dromiciops gliroides (Microbiotheria: Microbiotheriidae) in the deciduous forests of central Chile. Mammal Biol 70:376-380

Lopes G, Leiner NO (2014) Semelparity in a population of Gracilinanus agilis (Didelphimorphia: Didelphidae) inhabiting the Brazilian cerrado. Mammal Biol 80:1-6.

Mann G (1955) Monito del monte Dromiciops australis Phillipi. Inv Zool Chilenas 2:159-166

Martin G (2003) Nuevas localidades para marsupiales patagónicos (Didelphimorphia y Microbiotheria) en el noroeste de la provincia del Chubut, Argentina. Mastozool Neotrop 10:148-153

Martin GM (2010) Geographic distribution and historical occurrence of Dromiciops gliroides Thomas (Marsupialia, Microbiotheria). J Mammal 91:1025-1035

Muñoz-Pedreros A, Lang BK, Bretos M, Meserve PL (2005) Reproduction and development of Dromiciops gliroides (Marsupialia: Microbiotheriidae) in temperate rainforests of southern Chile. Gayana Zool 69:225-233

Nilsson MA, Arnason U, Spencer PBS, Janke A (2004) Marsupial relationships and a timeline for marsupial radiation in South Gondwana. Gene 340:189-196

Palma RE, Spotorno AE (1999) Molecular systematics of marsupials based on the rRNA 125 mitochondrial gene: the phylogeny of Didelphimorphia and of the living fossil microbiotheriid Dromiciops gliroides Thomas. Mol Phylogenet Evol 13:525-535

Patterson BD, Meserve PL, Lang BK (1990) Quantitative habitat associations of small mammals along an elevational transect in temperate rainforest of Chile. J Mammal 71:620-633

Pearson OP (1983) Characteristics of a mammalian fauna from forests in Patagonia, southern Argentina. J Mammal 64:476-492

Pearson AK, Pearson OP, Gomez IA (1994) Biology of the bamboo Chusquea culeou (Poaceae: Bambusoideae) in southern Argentina. Vegetation 111:93-126

Premoli AC, Kitzberger T, Veblen TT (2000) Isozyme variation and recent biogeographical history of the long-lived conifer Fitzroya cupressoides. J Biogeogr 27:251-260

Rapoport EH, Ladio A, Sanz E (2003) Plantas nativas comestibles de la Patagonia Andina Argentino Chilena. Parte II. Ediciones alternatura. Bariloche. Secretaría de Extensión Universitaria. Universidad Nacional del Comahue, Fundación Antorchas

Rodríguez-Cabal MA, Branch LC (2011) Influence of habitat factors on the distribution and abundance of a marsupial seed disperser. J Mammal 92:245-1252

Rodríguez-Cabal MA, Amico GC, Novaro AJ, Aizen MA (2008) Population characteristics of Dromiciops gliroides (Philippi, 1893), an endemic marsupial of the temperate forest of Patagonia. Mammal Biol 73:74-76

Sage RD, Pearson OP, Sanguinetti J, Pearson AK (2007) Ratada 2001: a rodent outbreak following the flowering of bamboo (Chusquea culeou) in southwestern Argentina. In: Kelt DA, Kaspin D (eds) The Quintessential 
Naturalist: Honoring the Life and Legacy of Oliver P. Pearson. University of California Press, USA, pp 177-224

Sanguinetti J, Contreras MR, Miño G, García L (2012) Nueva floración masiva de la Caña Colihue (Chusquea culeou) en el Parque Nacional Lanín y zonas adyacentes en Neuquén. Medidas de manejo, prevención y monitoreo, Informe a Parques Nacionales Argentina Diciembre de 2012

Smith AP, Broome $L$ (1992) The effects of season, sex and habitat on the diet of the mountain pygmypossum (Burramys parvus). Wildl Res 19:755-767.

Stannard HJ, Caton W, Old JM (2010) The diet of red-tailed phascogales (Phascogale calura) in a trial translocation at Alice Springs Desert Park, Northern Territory. J Zool 280:326-331. doi:10.1111/j.1469-7998.2009.00658.x Tyndale-Biscoe H (2005) Life of marsupials. CSIRO Publishing, Collingwood Vila A, Borelli L (2011) Cattle in the Patagonian forests: feeding ecology in Los Alerces National Reserve. Forest Ecol Manag 261:1306-1314

\section{Submit your manuscript to a SpringerOpen ${ }^{\circ}$} journal and benefit from:

- Convenient online submission

$\checkmark$ Rigorous peer review

- Immediate publication on acceptance

- Open access: articles freely available online

- High visibility within the field

- Retaining the copyright to your article

Submit your next manuscript at $\boldsymbol{\wedge}$ springeropen.com 